\title{
Fatigue Related Changes in Electromyographic Coherence between Homologous Vastus Lateralis Muscles
}

\author{
Andrius Šatas, Giedrė Jurgelaitienė, Albertas Skurvydas \\ Lithuanian Sports University, Kaunas, Lithuania
}

\begin{abstract}
Background. The aim of this study was to examine changes in the coherence between EMG signals of homologous quadriceps (vastus lateralis, $\mathrm{VL}$ ) muscles during in the beginning and the end of multiple sustained isometric bilateral contraction at maximal effort.

Methods. Twelve young healthy males participated in the study. The increase in coherence between EMG signals of homologous VL muscles in alpha band, beta band and gamma band was observed during the performance of sustained maximal bilateral isometric contraction with simultaneous decreases in bilateral force, CAR. EMG frequency and EMG amplitude.

Results. The increase in beta band coherence during sustained maximal isometric contraction was negatively correlated with the decrease in bilateral force, CAR, EMG amplitude and EMG frequency indicating the possible role of beta coherence to improve VL muscle activation efficiency during maximal bilateral isometric contraction and overcome the effects of VL muscle fatigue in young healthy males.
\end{abstract}

Keywords: muscle fatigue, EMG coherence, bilateral contraction, common drive.

\section{INTRODUCTION}

$\mathrm{M}$ uscle fatigue is an exercise-induced reduction in the muscle's capability to generate force (Gandevia, 2001). This reduction of force generation capability depends on the specifics of the task (Enoka \& Duchateau, 2008) and manifests as muscle force fluctuations (Hunter, Duchateau, \& Enoka, 2004), changes in motor unit recruitment (Carpentier, Duchateau, \& Hainaut, 2001), electromyographic (EMG) amplitude (Bernecke et al., 2017), motor unit (MU) discharge variability (Mottram, Maluf, Stephenson, Anderson, $\&$ Enoka, 2006) and decreases in discharge rates of MUs (Carpentier et al., 2001).

It was demonstrated that during bilateral movements the participating limbs do not move independently but influence each other (Boonstra et al., 2008). It has been suggested that bilateral coupling primarily stems from shared efferent information (Ridderikhoff, Peper, \& Beek, 2005). Muscles that are normally coactivated may share a common drive arising from branched presynaptic fibers or from presynaptic synchronization of lastorder inputs (Carr, Harrison, \& Stephens, 1994). Alternatively, bilateral coupling may arise from cortical neural crosstalk between bilateral motor areas through the corpus callosum (Oliveira, Gribova, Donchin, Bergman, \& Vaadia, 2001). It was shown that fatigue influences muscle coordination patterns between synergistic (Kattla \& Lowery, 2010) or homologous (Boonstra et al., 2008) muscles. EMG-EMG coherence is a tool for studying the effect of fatigue on muscle coordination (Danna-Dos Santos et al., 2010).

Coherence can be used as an index of muscle coordination between pairs of muscles by identifying the strength and periodicity of common frequency characteristics between two EMG signals (Farmer, 1998), and analysis of the coherence between electromyographic (EMG) signals could provide a means to examine the characteristics of common 
neural inputs to co-contracting muscles during voluntary contraction (Kattla \& Lowery, 2010). In addition, analysis of EMG-EMG coherence across muscles may provide insight into the temporal modulation of oscillatory activity at the cortical level and central nervous system control strategies for maintaining force output as muscle fatigues (Kattla \& Lowery, 2010).

The central nervous system exerts control over voluntary movements via the MUs of pertinent muscles and this control is considered oscillatory in nature (Farmer, 1999). The central nervous system oscillatory drives responsible for the coherent activity between two EMG signals are typically characterized within four different frequency bands. These include a low frequency 1-3 Hz oscillation, that may also be observed as the common modulation of motor-unit firing times known as 'common drive' (De Luca and Erim, 1994; Myers et al., 2004), oscillations at the frequency of physiological tremor or alpha range $(8-12 \mathrm{~Hz})$ (McAuley and Marsden, 2000) and within the beta $(15-30 \mathrm{~Hz})$ and gamma $(30-60 \mathrm{~Hz})$ frequency ranges (Conway et al., 1995; Mima and Hallett, 1999; Omlor et al., 2007). The similarity between EMG-EMG coherence and well-established corticomuscular encephalographic (EEG) and EMG coherence (Conway et al., 1995; Salenius et al., 1997) suggests that the common motoneuronal activity within $15-30 \mathrm{~Hz}$ frequency range is likely to be, at least partially, cortical in origin (Baker, Olivier, \& Lemon, 1997; Kattla \& Lowery, 2010).

EMG-EMG coherence analysis could be applied clinically in the investigation of movement disorders (Grosse, 2002). An abnormally large EMG-EMG coherence in the ipsilateral muscle pair (right wrist extensor and first dorsal interosseus muscle) of the upper extremity was observed in the patients with cortical myoclonus compared to healthy subjects (Grosse, 2002). In addition, atypical increase in alpha band EMG-EMG coherence and decrease in beta and gamma band coherence were observed in the presence of Parkinson's disease (Salenius, Avikainen, Kaakkola, Hari, \& Brown, 2002). Furthermore, it was demonstrated, that patients with upper limb dystonia show abnormal coherence between extensor carpi radialis and flexor carpi radialis over $1-12$ and $14-32 \mathrm{~Hz}$ frequency bands leading to the suggestion, that cortical drives may be responsible for the co-contraction of antagonistic muscles in this condition (Farmer et al., 1998). Alternatively, Kamp et al. (2013) demonstrated an increased corticomuscular coherence in beta band during isometric contraction of first dorsal interossei subjects aged above 40 years suggesting changes in beta band EMG-EMG coherence as an early neurophysiological marker of healthy aging.

A few studies have investigated changes in EMG coherence of homologous elbow flexors and extensors (Boonstra, Daffertshofer, van As, van der Vlugt, \& Beek, 2007) and synergistic finger muscles (Danna-Dos Santos et al., 2010; Kattla \& Lowery, 2010) during fatiguing submaximal isometric contractions. Boonstra et al. (2007) demonstrated presence of bilateral MU synchronization between homologous elbow flexors and extensors in $8-12 \mathrm{~Hz}$ frequency range during isometric force production task. This synchronization increased with fatigue and more strongly so for elbow extensor than for flexor muscles. Kattla and Lowery (2009) found that EMG-EMG coherence between an intrinsic and extrinsic index flexor muscle significantly increased in the 15-60 Hz frequency range when comparing pre- and post-fatigue EMG signals from an isometric index finger flexion task. Similarly, Danna-Dos Santos et al. (2010) found, that EMGEMG coherence in $0-35 \mathrm{~Hz}$ frequency range was significantly greater at the end than at the beginning of the fatiguing contraction during finger grasping task. However, synchronization across simultaneously active homologous vastus lateralis (VL) muscles during sustained maximal isometric contraction have not yet been examined. Results of Boonstra et al. 2007 demonstrated the presence of functional organization of common drive: the EMG-EMG coherence of flexor muscles of upper extremity were lower compared to extensor muscles, indicating that flexor muscles are controlled more independently than extensor muscles. In addition results of Volz et al. (2015) revealed differences in cortical motor control network suggesting, that the cortical control of upper limb movements are more lateralized compared to lower limb movements and implying stronger modulation of cortical control of hand movements ( i.e. fine-tuned grasping) compared to lower limb movements (locomotion, gait). Such differences in functional network configurations underlying lower limb and upper limb movements may impact changes in EMGEMG coherence (Volz et al., 2015).

The aim of this study was to examine changes in coupling between homologous quadriceps muscles during performance of sustained maximal isometric contraction of homologous VL muscles. 
Coherence between surface EMG signals from right $\mathrm{VL}$ and left $\mathrm{VL}$ were compared in the beginning and the end of sustained contraction. We predicted, that performance of sustained maximal isometric contraction will cause muscle fatigue which in turn will cause the decreases of muscle force, central activation ratio (CAR), EMG amplitude, EMG frequency as demonstrated in numerous studies (Bernecke et al., 2017). We have also expected that EMG-EMG coherence between simultaneously active homologous VL muscles will increase during performance of sustained contraction due to increase in common input to both muscles in order to facilitate activation of MUs and overcome the effects of fatigue (Boonstra et al., 2008).

\section{MATERIALS AND METHODS}

Participants. 12 young healthy males participated in the experiment (Table 1). The participants were physically active and participated in various sporting activities at a frequency of 2 to 3 times a week. All participants were nonsmokers, without any history of significant knee injury or surgery, pain during resisted knee extension, pain or instability during functional activities, fracture of the pelvis, femur, tibia, fibula, or patella within the past 2 years. All participants gave their informed consent, which was approved by the Human Research Ethics Committee. All procedures performed in studies involving human participants were in accordance with the ethical standards of the institutional and/or national research committee and with the 1964 Declaration of Helsinki and its later amendments or comparable ethical standards.

Table 1. Physical characteristics of the participants

\begin{tabular}{|c|c|}
\hline Characteristics & $\boldsymbol{n}=\mathbf{1 2}$ \\
\hline Age (year) & $22.4 \pm 1.6$ \\
\hline Height $(\mathrm{cm})$ & $179.4 \pm 7.6$ \\
\hline Weight $(\mathrm{kg})$ & $80.1 \pm 9.1$ \\
\hline BMI $\left(\mathrm{kg} / \mathrm{m}^{2}\right)$ & $23.8 \pm 2.1$ \\
\hline Body fat $(\%)$ & $12.33 \pm 2.4$ \\
\hline
\end{tabular}

Note. Values are expressed as means and SD, BMI - body mass index.

Experiment design. To attain a stable level of performance, one week before the experimental trials, participants attended a familiarization session during which they were introduced to the experimental apparatus and procedures for motor testing and trained to perform bilateral maximal voluntary contraction. All experimental sessions started at 12 p.m. Upon arrival to the laboratory, subjects were seated in custom chair, EMG and electrostimulation electrodes were attached to right and left vastus lateralis (VL) muscles. Knee angles of both legs were set at $60^{\circ}$ (full knee extension $=$ $180^{\circ}$ ). Each subject completed a sustained maximal isometric bilateral contraction (SC), contraction lasted 1 minute (Figure 1). A standard vocal encouragement was provided for the subjects by the same investigator during performance of bilateral isometric contraction in order to ensure maximal effort from the subjects. Electrical muscle stimulation (MS) was applied on $3^{\text {rd }}$ and $57^{\text {th }}$ seconds during $\mathrm{SC}$ in order to measure central activation ratio (CAR).

Bilateral force measurement and electrical stimulation. Subjects were seated in custom chair with adjustable lower limb attachments in upright position (hip angle $110^{\circ}$ ) and were tied up with waist and chest belts. The axis of rotation of the lower limb attachments were aligned with the axis of rotation of subject's knee joints. Subject's legs were secured to the lower limb attachment pads with ankle belts above lateral malleoli. The force of quadriceps of both legs was measured via LPU100 load cells (Transducer Techniques, Temecula, CA). The load cells were bolted to the bottom part of lower limb attachment and connected to the base of the chair by using rigid metal rod with ball joint. The angle between load cell and connecting rod was kept constant at $90^{\circ}$ to prevent non-axial loading. Load cells were tested with static weights and calibrated prior to experiments. The initial signal levels were set to zero to correct for the weight of each limb. Force signals were amplified (gain $=40)$, digitized online by BIOPAC MP150 data acquisition system (sampling rate - $1 \mathrm{kHz}$ ) and recorded simultaneously with Acqknowledge 4.1 software (BIOPAC Systems Inc., Goleta, CA). Subjects were required to keep their arms crossed in front of their chest.

Direct muscle stimulation was applied bilaterally using carbonized rubber electrodes (FIAB, Italy) covered with a thin layer of ECG gel (Ceracarta, Italy). The anode $(8 \times 24 \mathrm{~cm})$ was placed transversely across the width of the proximal portion of the quadriceps femoris and the cathode $(8 \times 12$ $\mathrm{cm}$ ) was placed on the distal portion of the muscle, above the patella. A high-voltage constant current stimulator (DS7, Digitimer Ltd, UK) was used to deliver electrical stimuli (monophasic square wave 
pulses; pulse width, $1 \mathrm{~ms}$ ). Tetanic pulse (2 pulses; $1 \mathrm{~ms}$ duration, $10 \mathrm{~ms}$ interpulse interval) was used for CAR calculation.

Electromyography recording. EMG activity was recorded from vastus lateralis of each leg. The skin was shaved and cleaned with alcohol to reduce impedance below $5 \mathrm{k} \Omega$ between skin and electrodes. EMG signals were obtained by using two silver chloride electrodes $(26 \mathrm{~mm}$ diameter; center-to-center distance $-2,5 \mathrm{~cm}$ ) attached to the proximal and distal parts of the bellies of the VL muscles. The common reference electrodes were placed under the knee caps. EMG signals were amplified (gain $=1000)$, band-pass filtered $(10 \mathrm{~Hz}-$ $5 \mathrm{kHz}$ ), digitized online (sampling rate $-2 \mathrm{kHz}$ ) and recorded simultaneously with Acqknowledge 4.1 software (BIOPAC Systems Inc., Goleta, CA).

Data analysis. Coherence calculation. The data analysis was done using custom MATLAB scripts (MathWorks, Inc., USA). EMG data were filtered off-line (second-order bidirectional Butterworth band-pass filter: 5-300 Hz), full-wave rectified using the Hilbert transform (Myers et al., 2003). For estimating the strength of interlimb coordination, we analyzed coherence between EMG outputs from the two muscles (left VL and right VL) using magnitude-squared coherence (Bendat \& Piersol, 2010). This coherence analysis can quantify the cross-correlation between pairs of force signals within a trial at each frequency of signal. The coherence values ranged from 0 to 1 , and values close to 1 indicated a high correlation between the two legs at each frequency indicating better interlimb coordination patterns.

$$
C_{x y}(f)=\frac{\left|P_{x y}(f)\right|^{2}}{P_{x x}(f) P_{y y}(f)}
$$

Where $\mathrm{x}$ is left force signals and $\mathrm{y}$ is right force signals. Pxy (f) is the cross-spectral density between left and right force signals. Pxx (f) and Pyy (f) are the autospectral density of left and right force signals respectively. The coherence was calculated for a segment length of 4,096 samples $50 \%$ overlap, using a Hanning window. Two $10 \mathrm{~s}$ segments from EMG data $\left(2-12 \mathrm{~s}: \mathrm{SC}_{\text {begin }} ; 48-58 \mathrm{~s}\right.$ : $\mathrm{SC}_{\text {end }}$ ) from sustained contraction were selected for analysis (Figure 1). The confidence level $(\alpha=0.05)$ was estimated by performing a surrogate data analysis approach. Surrogate series were generated for each EMG signal $\mathrm{x}(\mathrm{t})$ by calculating the Fourier transform, independently shuffling the phase components, and calculating the inverse Fourier transform (Faes, Pinna, Porta, Maestri, \& Nollo, 2004). The area of significant coherence was calculated for alpha $(8-15 \mathrm{~Hz})$, beta $(15-35 \mathrm{~Hz})$, and gamma $(35-60 \mathrm{~Hz})$ frequency bands for both $\mathrm{SC}_{\text {begin }}$ and $\mathrm{SC}_{\text {end }}$ data segments and used for statistical analysis.

Bilateral force and central activation ratio. The bilateral force and CAR were calculated during the sustained fatiguing contraction for $1 \mathrm{~s}$ duration epochs for $\mathrm{SC}_{\text {begin }}\left(3^{\text {rd }}\right.$ second) and $\mathrm{SC}_{\text {end }}\left(57^{\text {th }}\right.$ second) data segments and each muscle (right VL and left VL) (Figure 1). The force values of right
Figure 1. Experimental protocol. Representation of sustained maximal bilateral isometric contraction of homologous vastus lateralis muscles and data segments used for analysis
Notes. MS - electrical muscle stimulation, CAR - central activation ratio, EMG RMS electromyographic amplitude, EMG meanF - electromyographic mean frequency, $\mathrm{SC}_{\text {begin }}$ - beginning (2-12 s) of sustained contraction, $\mathrm{SC}_{\text {end }}$ ending of sustained contraction (48-58 s).

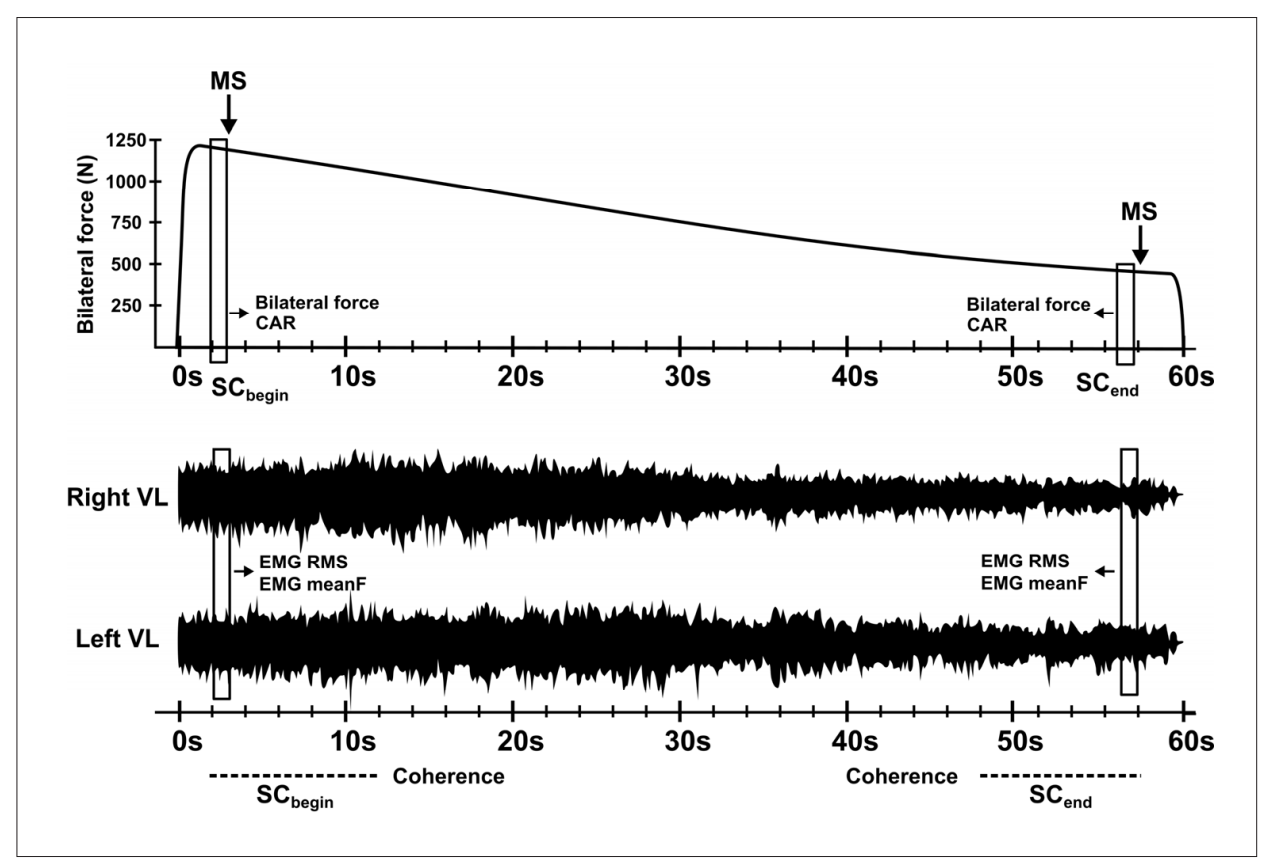


$\mathrm{VL}$ and left VL were summed and normalized in respect to initial values for each subject and each data segment.

EMG RMS amplitude and mean frequency. The root mean square (RMS) value of the EMG signal and the mean frequency (meanF) of the EMG power spectrum were calculated using builtin MATLAB functions (MathWorks, Inc., USA). during the sustained fatiguing contraction for $1 \mathrm{~s}$ duration epochs for $\mathrm{SC}_{\text {begin }}\left(3^{\text {rd }}\right.$ second) and $\mathrm{SC}_{\text {end }}$ (57 th second) data segments and each muscle (right $\mathrm{VL}$ and left $\mathrm{VL}$ ). The values of right $\mathrm{VL}$ and left $\mathrm{VL}$ were averaged and normalized in respect to initial values for each subject and each data segment $\left(\mathrm{SC}_{\text {begin }}\right.$ and $\left.\mathrm{SC}_{\text {end }}\right)$ (Figure 1).

Statistical analysis. Data are reported as means \pm SD. The data were tested for normal distribution using the Kolmogorov-Smirnov test, and all were found to be normally distributed. The area of the EMG-EMG coherence values that lay above the $95 \%$ significance level in each of the alpha $(8-15 \mathrm{~Hz})$, beta $(15-35 \mathrm{~Hz})$ and gamma
(35-60 Hz) frequency bands, bilateral force, EMG RMS, EMG meanF during the $\mathrm{SC}_{\text {begin }}$ and $\mathrm{SC}_{\text {end }}$ of the fatiguing contraction, were analyzed for statistical significance using paired t-tests. Pearson correlation coefficient (r) was used to identify relationship between the changes of bilateral force, CAR, EMG RMS, EMG meanF, alpha coherence, beta coherence. The level of significance was set at $\mathrm{p}<0.05$. All statistical analyses were performed using SPSS (version 21.0; IBM Corp., Armonk, NY, USA).

\section{RESULTS}

Data on changes in magnitude squared coherence between EMG signals of right VL and left VL is presented in Figure 2. Magnitude squared coherence between EMG signals of right VL and left VL significantly increased during $\mathrm{SC}_{\text {end }}$, compared to $\mathrm{SC}_{\text {begin }}$ in alpha band $(t=-3.0$, $p=.01)$, beta band $(t=-3.4, p=.006)$ and gamma band $(t=-2.3, p=.04)$.
Figure 2. Coherence between right and left vastus lateralis EMG signals at the beginning $\left(\mathrm{SC}_{\mathrm{begin}}\right.$, solid line $)$ and ending $\left(\mathrm{SC}_{\mathrm{end}}\right.$ dashed line) of sustained maximal isometric contraction (A). Mean and standard deviation of the integral of the significant coherence in the beginning $\left(\mathrm{SC}_{\text {begin }}\right)$ and ending $\left(\mathrm{SC}_{\mathrm{end}}\right)$ of sustained maximal isometric contraction $(\mathrm{B})$
A
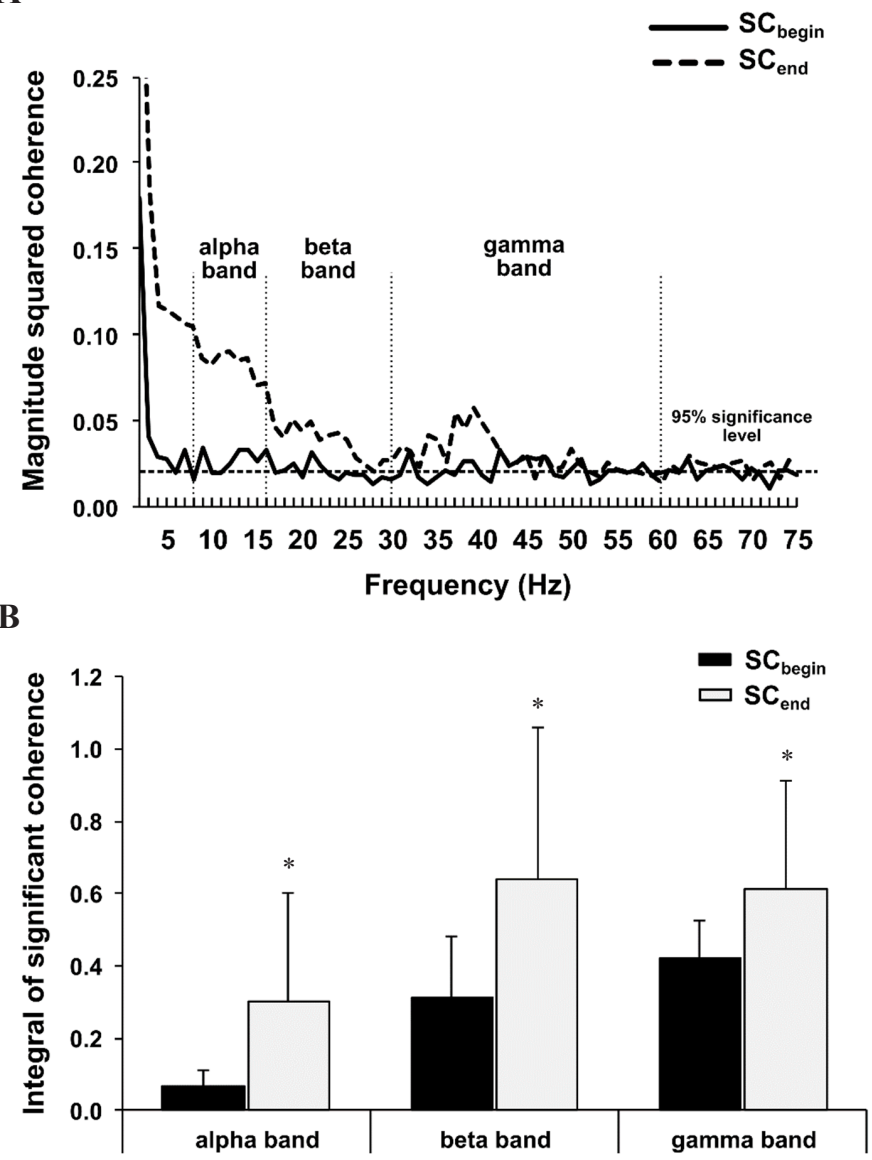
Data on initial values of bilateral force, CAR, EMG RMS, EMG meanF is presented in Table 2. There was a significant decrease in bilateral force $(t=18.6, p=.0001), \mathrm{CAR}(t=3.7, p=.03), \mathrm{EMG}$ RMS $(t=9.6, p=.001)$, EMG meanF $(t=9.4$, $p=.001$ ) during $\mathrm{SC}_{\text {end }}$, compared to $\mathrm{SC}_{\text {begin }}$ (Table 3).

Significant positive correlations were found between changes in bilateral force and alpha coherence $(r=.78, p=.002)$ (Figure $3 \mathrm{~A}$ ); also between changes in CAR and alpha coherence $(r=.74, p=.004)$ (Figure $3 \mathrm{~B})$.

Significant negative correlations were found between changes in beta coherence and bilateral force $(r=-.60, p=.03)$ (Figure $4 \mathrm{~A}$ ); beta coherence and CAR $(r=-.62, p=.02)$ (Figure 4 B); beta coherence and EMG meanF $(r=-.68$, $p=.01$ ) (Figure $4 \mathrm{C}$ ); beta coherence and EMG RMS $(r=-.64, p=.02)$ (Figure $4 \mathrm{D})$.
Table 2. Baseline values

Note. CAR - central activation ratio, EMG MeanF - electromyographic mean frequency, EMG RMS - electromyographic amplitude.

\begin{tabular}{|l|c|c|c|c|}
\hline & $\begin{array}{c}\text { Bilateral Force } \\
(\mathbf{N})\end{array}$ & $\begin{array}{c}\text { CAR } \\
(\mathbf{\%})\end{array}$ & $\begin{array}{c}\text { EMG MeanF } \\
(\mathbf{H z})\end{array}$ & $\begin{array}{c}\text { EMG RMS } \\
(\mathbf{m V})\end{array}$ \\
\hline Mean & 1210.75 & 97.82 & 144.19 & 0.51 \\
\hline SD & \pm 270.66 & \pm 1.96 & \pm 21.89 & \pm 0.14 \\
\hline
\end{tabular}

Table 3. Effect of Sustained Maximal Isometric Bilateral Contraction of Homologous Vastus Lateralis Muscles on Bilateral Force, CAR, EMG Mean Frequency, EMG Amplitude. * $p<.05$ compared with $\mathrm{SC}_{\text {begin }}$

Note. Values are expressed as means and SD, CAR - central activation ratio, EMG MeanF - electromyographic mean frequency, EMG RMS - electromyographic amplitude.

\begin{tabular}{|l|c|c|}
\hline & SC $_{\text {begin }}$ & SC $_{\text {end }}$ \\
\hline Bilateral force (\%) & $100.00 \pm 0.00$ & $46.10 \pm 10.44^{*}$ \\
\hline CAR (\%) & $97.82 \pm 1.96$ & $91.82 \pm 5.85^{*}$ \\
\hline EMG MeanF (\%) & $100.00 \pm 0.00$ & $83.27 \pm 6.40^{*}$ \\
\hline EMG RMS (\%) & $100.00 \pm 0.00$ & $61.66 \pm 14.30^{*}$ \\
\hline
\end{tabular}

Figure 3. Correlations between changes in EMG-EMG alphaband (8-15 Hz) coherence of homologous vastus lateralis muscles and changes in bilateral force (A), central activation ratio (B), EMG frequency (C) and EMG amplitude (D)

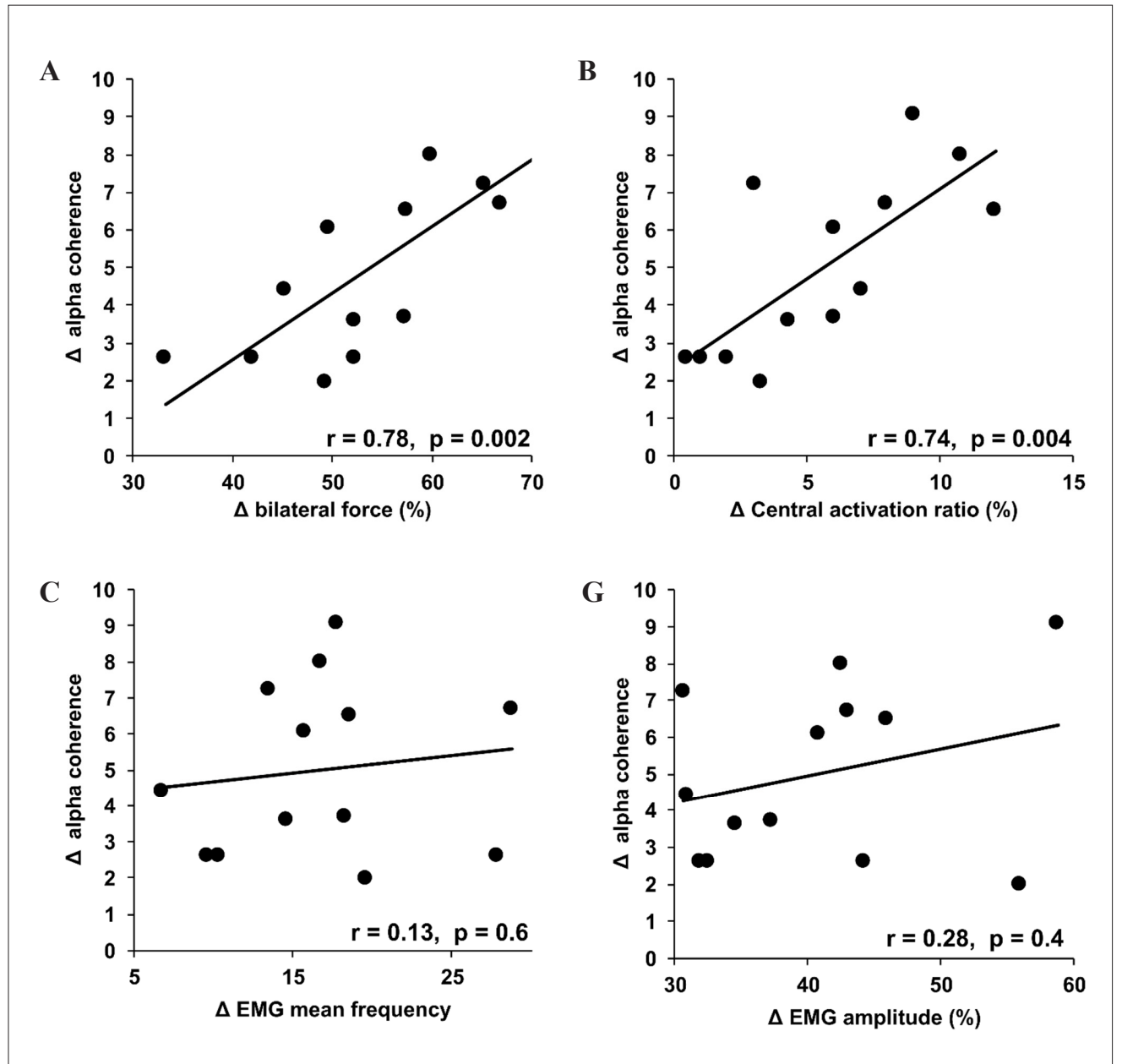


A

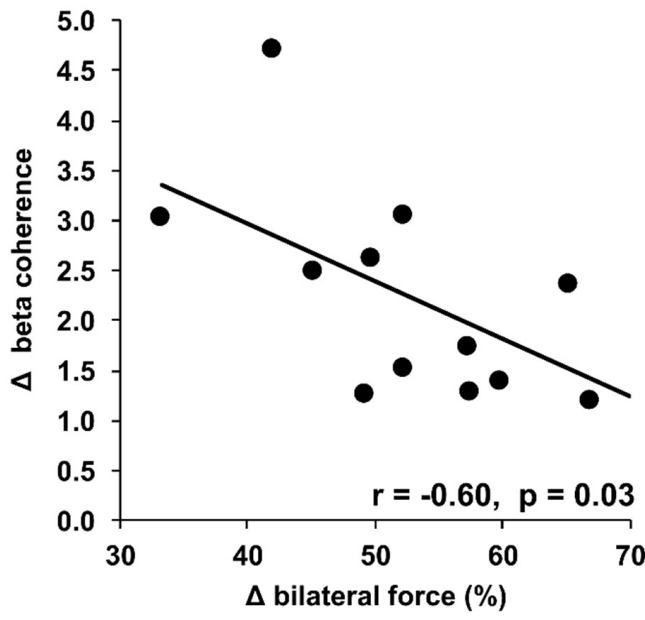

C

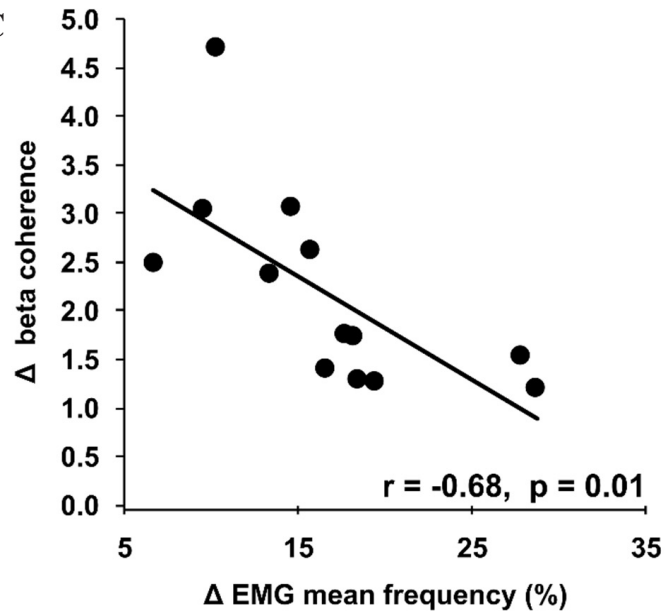

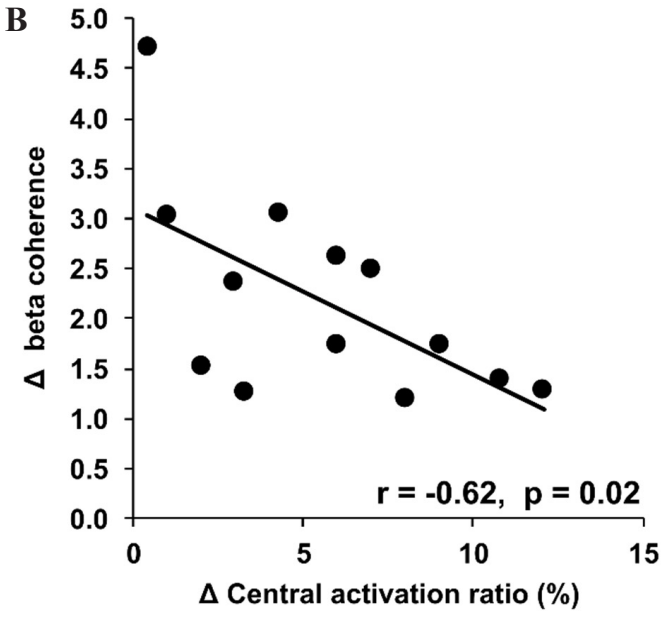

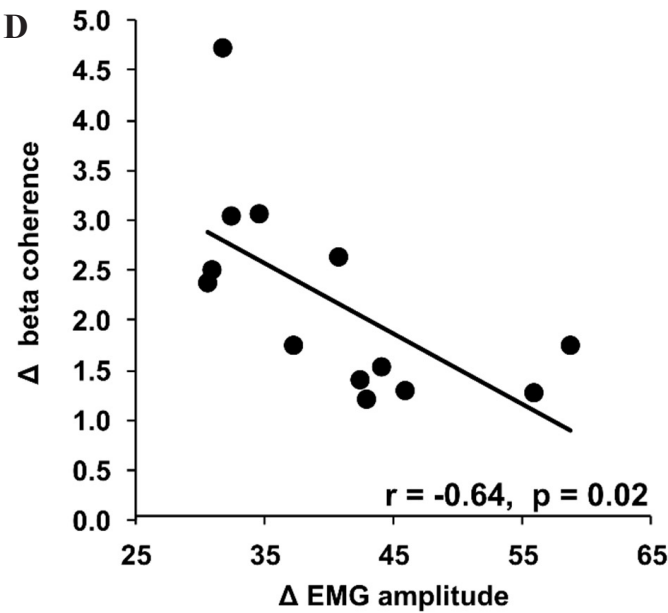

Figure 4. Correlations between changes in EMG-EMG beta band (15-35Hz) coherence of homologous vastus lateralis muscles and changes in bilateral force (A), central activation ratio (B), EMG frequency (C) and EMG amplitude (D)

\section{DISCUSSION}

The aim of this study was to examine changes in the coherence between EMG signals of homologous VL muscles during in the beginning and the end of multiple sustained isometric contraction. The results of this study indicated an increase in coherence between EMG signals of homologous VL muscles in alpha band, beta band and gamma band during performance sustained maximal bilateral isometric contraction with simultaneous decreases in bilateral force, CAR. EMG frequency and EMG amplitude. In addition, the increase in beta band coherence during sustained maximal isometric contraction was negatively correlated with the decrease in bilateral force, CAR, EMG amplitude and EMG frequency.

As expected, bilateral muscle force declined during sustained maximal isometric contraction. It was reported, that decreased central activation of motor units and possibly impaired action potential propagation within the muscle fibers is responsible for force decline during prolonged muscle contraction (Arendt-Nielsen, Mills, \& Forster, 1989; Broman, Bilotto, \& De Luca, 1985). In agreement with previous studies (Brazaitis et al., 2012; KentBraun, 1999) we observed the decline of CAR at the end of sustained maximal isometric contraction $\left(\mathrm{SC}_{\text {end }}\right)$ (Table 3). Another possible mechanism of progressive force decline during sustained contraction is accumulation of inorganic phosphate ions (Pi) caused by the breakdown of phosphocreatine together with an increase in adenosine diphosphate (ADP) and hydrogen ion $(\mathrm{H}+)$ concentrations can lead to a decreased force production in fatigue (Allen, Lamb, \& Westerblad, 2008).

During the sustained maximal fatiguing contraction, EMG amplitude and the EMG median 
frequency progressively decreased (Table 3). These changes are typical of myoelectric manifestations of fatigue during maximal isometric contraction and confirm the progression of muscle fatigue during the sustained contraction (Bernecke et al., 2017). The decrease in the mean frequency observed is primarily due to the reduction in motor neuron firing rate (Bigland-Ritchie, Johansson, Lippold, Smith, \& Woods, 1983), while the decrease in the RMS amplitude reflects decreasing motor-unit recruitment and firing rates due to central fatigue (BiglandRitchie \& Woods, 1984). The changes in bilateral force, CAR, EMG amplitude and EMG frequency confirm the progression of muscle fatigue.

In the present study, significant coherence between EMG signals from the right VL and left VL muscles was observed in alpha, beta and gamma frequency bands during sustained maximal isometric contraction (Figure $2 \mathrm{~A}$ ). There was a significant increase in alpha coherence during sustained maximal isometric contraction (Figure 2 B). Most likely, the increase in alpha coherence reflected tremor activity, which has been studied extensively (McAuley \& Marsden, 2000). Tremor around $10 \mathrm{~Hz}$ is well-documented and typically referred to as physiological or central tremor (McAuley, Rothwell, \& Marsden, 1997). It was demonstrated, that physiological tremor is affected by muscle fatigue in that the tremor strength increases during isometric contraction at high force levels (Ebenbichler et al., 2000). A possible explanation for the bilateral coherence in alpha band is the phenomenon of motor irradiation (an increase of bilateral co-activation during unilateral contraction at high force) (Arányi \& Rösler, 2002).

The decrease in bilateral force was associated with an increase of the alpha coherence, i.e. alpha coherence increased during sustained maximal isometric contraction (Figure $3 \mathrm{~A}$ ). This finding is consistent with the results of Christou et al. (2007), which demonstrated that increased MU synchronization is fatigue dependent.

Presence of EMG-EMG coherence in beta frequency band was demonstrated between different hand muscles using rectified surface EMGs (Kilner et al., 1999). Results of Baker et al. (1997) have demonstrated, that changes in beta band EMG-EMG coherence in primates reflects changing levels of synchronous drive from the corticospinal system and exhibits a similar task-dependence to corticomuscular coupling as estimated using EEG-EMG coherence. There was a significant increase in EMG-EMG beta band coherence of right VL and left VL muscles during sustained maximal isometric contraction (Figure 2 B). Similar increases in beta coherence was demonstrated between MEG-EMG signals hand muscles in response to fatiguing contraction (Tecchio et al., 2006). If EMG-EMG coherence within the beta and gamma frequency bands reflects an increase in synchronized corticospinal common inputs to the motoneuron pool across the muscles, one possibility may be that synchronization of motoneuron inputs may help to overcome reduced excitability of the motoneuron pool during fatigue (Kattla \& Lowery, 2010). Results of Murthy and Fetz (1994) indicated, that synchronized inputs of the sensorimotor cortex are more effective at recruiting motoneurons than asynchronous inputs. In addition, Kilner et al. (Baker, Kilner, Pinches, $\&$ Lemon, 1999) proposed, that the functional role of MEG-EMG coherence might be to improve the efficiency with which motor neurons are recruited. Furthermore, Danna-Dos Santos et al. (2010) proposed role of coherence as a means to efficiently recruit motor neurons for maintaining the target force during fatiguing contraction, because the goal of generating a constant force has to be maintained despite a reduction in motor unit discharge rate through the recruitment of additional motor units (Mottram et al., 2006). In agreement with previous statements our results demonstrated that the increase in beta band coherence during sustained maximal isometric contraction was negatively correlated with the decrease in bilateral force, CAR, EMG amplitude and EMG frequency (Figure 4), indicating higher sustained maximal isometric contraction efficiency in the presence of higher beta band coherence.

There was a significant increase in EMGEMG gamma band coherence of right VL and left VL muscles during sustained maximal isometric contraction (Figure 2 B). It has been proposed that gamma oscillations may indicate binding of functionally associated cortical elements such as visual attention, motor planning and cognition (Brown, 2000) in order to maintain force output as muscle fatigue (Kattla \& Lowery, 2010).

There are several limitations in our study. Firstly, the generalizability of the results is limited by the fact that the sample consisted of young men. Kamp et al. (2013) demonstrated an increased corticomuscular coherence in beta band during isometric contraction of first dorsal interossei subjects aged above 40 years possibly reflecting an early neurophysiological marker of seniority. 
In addition to our knowledge, there are no studies on sex-related differences in corticomuscular or EMG-EMG coherence during prolonged isometric contraction. Thus, it would be beneficial replicate this research in women and elderly subjects.

\section{CONCLUSION}

In conclusion, we observed an increase in coherence between EMG signals of homologous VL muscles in alpha band, beta band and gamma band during performance sustained maximal bilateral isometric contraction with simultaneous decreases in bilateral force, CAR. EMG frequency and EMG amplitude. An important finding was that the increase in beta band coherence during sustained maximal isometric contraction was negatively correlated with the decrease in bilateral force, CAR, EMG amplitude and EMG frequency suggesting the role of beta coherence to improve VL muscle activation efficiency during maximal bilateral isometric contraction of VL muscles and overcome the effects of fatigue in young healthy males.

Conflict of Interest. The authors declare that they have no conflict of interest.

\section{REFERENCES}

Allen, D. G., Lamb, G. D., \& Westerblad, H. (2008). Skeletal muscle fatigue: Cellular mechanisms. Physiological Reviews, 88(1), 287-332. https://doi. org/10.1152/physrev.00015.2007

Arányi, Z., \& Rösler, K. M. (2002). Effort-induced mirror movements. A study of transcallosal inhibition in humans. Experimental Brain Research, 145(1), 76-82. https://doi.org/10.1007/s00221-002-1101-1

Arendt-Nielsen, L., Mills, K. R., \& Forster, A. (1989). Changes in muscle fiber conduction velocity, mean power frequency, and mean EMG voltage during prolonged submaximal contractions. Muscle \& Nerve, 12(6), 493-497. https://doi.org/10.1002/mus.880120610

Baker, S. N., Kilner, J. M., Pinches, E. M., \& Lemon, R. N. (1999). The role of synchrony and oscillations in the motor output. Experimental Brain Research, 128(1), 109-117. https://doi.org/10.1007/s002210050825

Baker, S. N., Olivier, E., \& Lemon, R. N. (1997). Coherent oscillations in monkey motor cortex and hand muscle EMG show task-dependent modulation. The Journal of Physiology, 501(1), 225-241. https://doi. org/10.1111/j.1469-7793.1997.225bo.x

Bendat, J. S., \& Piersol, A. G. (2010). Random Data: Analysis and Measurement Procedures (4 edition). Hoboken, N.J: Wiley.

Bernecke, V., Pukenas, K., Daniuseviciute, L., Baranauskiene, N., Paulauskas, H., Eimantas, N., \& Brazaitis, M. (2017). Sex-specific reliability and multidimensional stability of responses to tests assessing neuromuscular function. HOMO, 68(6), 452-464. https://doi.org/10.1016/j.jchb.2017.10.004

Bigland-Ritchie, B., Johansson, R., Lippold, O. C., Smith, S., \& Woods, J. J. (1983). Changes in motoneurone firing rates during sustained maximal voluntary contractions. The Journal of Physiology, 340(1), 335346. https://doi.org/10.1113/jphysiol.1983.sp014765

Bigland-Ritchie, B., \& Woods, J. J. (1984). Changes in muscle contractile properties and neural control during human muscular fatigue. Muscle \& Nerve, 7(9), 691699. https://doi.org/10.1002/mus. 880070902
Boonstra, T. W., Daffertshofer, A., van As, E., van der Vlugt, S., \& Beek, P. J. (2007). Bilateral motor unit synchronization is functionally organized. Experimental Brain Research, 178(1), 79-88. https://doi.org/10.1007/ s00221-006-0713-2

Boonstra, T. W., Daffertshofer, A., van Ditshuizen, J. C., van den Heuvel, M. R. C., Hofman, C., Willigenburg, N. W., \& Beek, P. J. (2008). Fatigue-related changes in motor-unit synchronization of quadriceps muscles within and across legs. Journal of Electromyography and Kinesiology, 18(5), 717-731. https://doi.org/10.1016/j. jelekin.2007.03.005

Brazaitis, M., Skurvydas, A., Pukenas, K., Daniusevičiūtè, L., Mickevičienè, D., \& Solianik, R. (2012). The effect of temperature on amount and structure of motor variability during 2-minute maximum voluntary contraction. Muscle \& Nerve, 46(5), 799-809. https://doi.org/10.1002/mus.23397

Broman, H., Bilotto, G., \& De Luca, C. J. (1985). Myoelectric signal conduction velocity and spectral parameters: Influence of force and time. Journal of Applied Physiology, 58(5), 1428-1437. https://doi. org/10.1152/jappl.1985.58.5.1428

Brown, P. (2000). Cortical drives to human muscle: The Piper and related rhythms. Progress in Neurobiology, 60(1), 97-108.

Carpentier, A., Duchateau, J., \& Hainaut, K. (2001). Motor unit behaviour and contractile changes during fatigue in the human first dorsal interosseus. The Journal of Physiology, 534(Pt 3), 903-912. https://doi. org/10.1111/j.1469-7793.2001.00903.x

Carr, L. J., Harrison, L. M., \& Stephens, J. A. (1994). Evidence for bilateral innervation of certain homologous motoneurone pools in man. The Journal of Physiology, 475(2), 217-227. https://doi.org/10.1113/jphysiol.1994. sp020063

Christou, E. A., Rudroff, T., Enoka, J. A., Meyer, F., \& Enoka, R. M. (2007). Discharge rate during low-force isometric contractions influences motor unit coherence below $15 \mathrm{~Hz}$ but not motor unit synchronization. 
Experimental Brain Research, 178(3), 285-295. https:// doi.org/10.1007/s00221-006-0739-5

Danna-Dos Santos, A., Poston, B., Jesunathadas, M., Bobich, L. R., Hamm, T. M., \& Santello, M. (2010). Influence of Fatigue on Hand Muscle Coordination and EMG-EMG Coherence During Three-Digit Grasping. Journal of Neurophysiology, 104(6), 3576-3587. https:// doi.org/10.1152/jn.00583.2010

Ebenbichler, G. R., Kollmitzer, J., Erim, Z., Löscher, W. N., Kerschan, K., Posch, M., ... Bochdansky, T. (2000). Loaddependence of fatigue related changes in tremor around $10 \mathrm{~Hz}$. Clinical Neurophysiology: Official Journal of the International Federation of Clinical Neurophysiology, 111(1), 106-111.

Enoka, R. M., \& Duchateau, J. (2008). Muscle fatigue: What, why and how it influences muscle function. The Journal of Physiology, 586(Pt 1), 11-23. https://doi. org/10.1113/jphysiol.2007.139477

Faes, L., Pinna, G. D., Porta, A., Maestri, R., \& Nollo, G. (2004). Surrogate data analysis for assessing the significance of the coherence function. IEEE Transactions on Biomedical Engineering, 51(7), 11561166. https://doi.org/10.1109/TBME.2004.827271

Farmer, S. F. (1998). Rhythmicity, synchronization and binding in human and primate motor systems. The Journal of Physiology, 509(Pt 1), 3-14. https://doi. org/10.1111/j.1469-7793.1998.003bo.x

Farmer, S. F., Sheean, G. L., Mayston, M. J., Rothwell, J. C., Marsden, C. D., Conway, B. A., ... Stephens, J. A. (1998). Abnormal motor unit synchronization of antagonist muscles underlies pathological co-contraction in upper limb dystonia. Brain: A Journal of Neurology, 121 ( Pt 5), 801-814. https://doi.org/10.1093/brain/121.5.801

Gandevia, S. C. (2001). Spinal and supraspinal factors in human muscle fatigue. Physiological Reviews, 81(4), 17251789. https://doi.org/10.1152/physrev.2001.81.4.1725

Grosse, P. (2002). EEG-EMG, MEG-EMG and EMGEMG frequency analysis: Physiological principles and clinical applications. Clinical Neurophysiology, 113(10), 1523-1531. https://doi.org/10.1016/S13882457(02)00223-7

Hunter, S. K., Duchateau, J., \& Enoka, R. M. (2004). Muscle fatigue and the mechanisms of task failure. Exercise and Sport Sciences Reviews, 32(2), 44-49.

Kamp, D., Krause, V., Butz, M., Schnitzler,A., \& Pollok, B. (2013). Changes of cortico-muscular coherence: An early marker of healthy aging? $A G E, 35(1), 49-58$. https://doi. org/10.1007/s11357-011-9329-y

Kattla, S., \& Lowery, M. M. (2010). Fatigue related changes in electromyographic coherence between synergistic hand muscles. Experimental Brain Research, 202(1), 8999. https://doi.org/10.1007/s00221-009-2110-0

Kent-Braun, J. A. (1999). Central and peripheral contributions to muscle fatigue in humans during sustained maximal effort. European Journal of Applied Physiology and Occupational Physiology, 80(1), 57-63. https://doi.org/10.1007/s004210050558
Kilner, J. M., Baker, S. N., Salenius, S., Jousmäki, V., Hari, R., \& Lemon, R. N. (1999). Task-dependent modulation of $15-30 \mathrm{~Hz}$ coherence between rectified EMGs from human hand and forearm muscles. The Journal of Physiology, 516(Pt 2), 559-570. https://doi. org/10.1111/j.1469-7793.1999.0559v.x

McAuley, J. H., \& Marsden, C. D. (2000). Physiological and pathological tremors and rhythmic central motor control. Brain: A Journal of Neurology, 123(Pt 8), 15451567. https://doi.org/10.1093/brain/123.8.1545

McAuley, J. H., Rothwell, J. C., \& Marsden, C. D. (1997). Frequency peaks of tremor, muscle vibration and electromyographic activity at $10 \mathrm{~Hz}, 20 \mathrm{~Hz}$ and 40 $\mathrm{Hz}$ during human finger muscle contraction may reflect rhythmicities of central neural firing. Experimental Brain Research, 114(3), 525-541. https://doi.org/10.1007/ p100005662

Mottram, C. J., Maluf, K. S., Stephenson, J. L., Anderson, M. K., \& Enoka, R. M. (2006). Prolonged Vibration of the Biceps Brachii Tendon Reduces Time to Failure When Maintaining Arm Position with a Submaximal Load. Journal of Neurophysiology, 95(2), 1185-1193. https://doi.org/10.1152/jn.00807.2005

Murthy, V. N., \& Fetz, E. E. (1994). Effects of input synchrony on the firing rate of a three-conductance cortical neuron model. Neural Computation, 6(6), 11111126. https://doi.org/10.1162/neco.1994.6.6.1111

Myers, L. J., Lowery, M., O’Malley, M., Vaughan, C. L., Heneghan, C., St Clair Gibson, A., ... Sreenivasan, R. (2003). Rectification and non-linear pre-processing of EMG signals for cortico-muscular analysis. Journal of Neuroscience Methods, 124(2), 157-165.

Oliveira, S. C. de, Gribova, A., Donchin, O., Bergman, H., $\&$ Vaadia, E. (2001). Neural interactions between motor cortical hemispheres during bimanual and unimanual arm movements. European Journal of Neuroscience, 14(11), 1881-1896. https://doi.org/10.1046/j.0953816x.2001.01801.x

Ridderikhoff, A., Peper, C. (Lieke) E., \& Beek, P. J. (2005). Unraveling interlimb interactions underlying bimanual coordination. Journal of Neurophysiology, 94(5), 31123125. https://doi.org/10.1152/jn.01077.2004

Salenius, S., Avikainen, S., Kaakkola, S., Hari, R., \& Brown, P. (2002). Defective cortical drive to muscle in Parkinson's disease and its improvement with levodopa. Brain: A Journal of Neurology, 125(Pt 3), 491-500. https://doi.org/10.1093/brain/awf042

Tecchio, F., Porcaro, C., Zappasodi, F., Pesenti, A., Ercolani, M., \& Rossini, P. M. (2006). Cortical shortterm fatigue effects assessed via rhythmic brain-muscle coherence. Experimental Brain Research, 174(1), 144151. https://doi.org/10.1007/s00221-006-0432-8

Volz, L. J., Eickhoff, S. B., Pool, E.-M., Fink, G. R., \& Grefkes, C. (2015). Differential modulation of motor network connectivity during movements of the upper and lower limbs. NeuroImage, 119, 44-53. https://doi. org/10.1016/j.neuroimage.2015.05.101 